\title{
MUJERES, MÚSICA Y LIDERAZGO
}

\section{WOMEN, MUSIC AND} LEADERSHIP

\author{
SANDRA SOLER CAMPO
}

\footnotetext{
${ }^{1}$ Universidad de Barcelona, España. Correo electrónico: sandra.soler@ub.edu
}

\begin{abstract}
Resumen
No podemos entender el ámbito de la creación artística sin la labor desempeñada por las mujeres. A pesar de que a lo largo de la historia muchas de ellas han sido silenciadas e incluso olvidadas. El camino a seguir (además de recuperar nuestro pasado histórico y premiar la trayectoria de todas las mujeres artistas olvidadas) es aquél que haga posible alcanzar el principio de igualdad sexo-genérica y que, por lo tanto, además de ofrecer un mayor número de oportunidades, pueda, a la vez, abordar el problema social y cultural con el objetivo de no volver a repetir la misma situación tan desfavorecedora para el género femenino.

Durante varios siglos la práctica de la enseñanza musical se ha ido convirtiendo en una práctica fundamentalmente femenina, no obstante, los líderes en este sector son los hom-
\end{abstract}


bres. De esta manera, las actividades que exigen liderazgo acostumbran a tener mayor presencia masculina, mientras que las mujeres en su mayoría dirigen corales o enseñan en las escuelas, y quienes dirigen grandes agrupaciones orquestales son los hombres.

Palabras clave: mujeres, música, liderazgo, historia, género

\begin{abstract}
We cannot understand the realm of artistic creation without the work of women. Although throughout history many of them have been silenced and even forgotten, the way forward (besides recovering our historical past and rewarding the trajectory of all forgotten women artists) is one that makes it possible to reach the beginning of equality gender-generic in addition to offering a greater number of opportunities, it can at the same time address the social and cultural problem with the objective of not repeating the same situation so disfavouring to the female gender.

For many centuries, the practice of music education has been a fundamentally feminine practice. However, the leaders in this sector are men. In activities that demand leadership, they tend to have a greater male presence. Thus, while women are the majority directing choirs or teaching in a school, those who lead large orchestral groups are men.
\end{abstract}




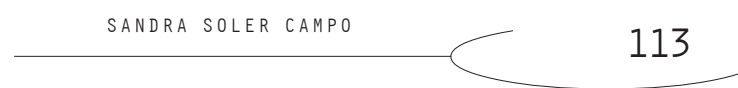

Keywords: women, music, leadership, history, gender

RECEPCIÓN: 31 DE JULIO DE 2018/ACEPTACIÓN: 16 DE OCTUBRE DE 2018

\section{INTRODUCCIÓN}

El plano musical, el campo compositivo, el de la in-

terpretación y, sobre todo, el de la dirección orquestal, han estado tradicionalmente dotados de un mayor estado de poder. Por ello, el acceso de la mujer a estos ámbitos ha sido muy restringido y en determinados periodos inexistente.

El interés por querer recuperar a las mujeres dentro del ámbito, (y cuando se hace mención de mujeres, la referencia es a su obra y aportación artística a la historia), se debe a la invisibilidad de muchas de ellas. Para los estudiantes de música y docentes, han sido muy pocas las referencias que se han tenido de mujeres. En manuales de música, por ejemplo, o a la hora de conocer los métodos compositivos, aspectos sociales y características musicales de cada periodo musical (Barroco, Clásico, Romanticismo...). A pesar de que cada vez podemos encontrar más editoriales que publican obras creadas por mujeres, tanto a nivel sonoro como en partitura, queda todavía una gran labor por hacer. También es necesario que se dejen de elaborar manuales y/o materiales didácticos que no recojan la vida y obra de artistas que forman parte de nuestro legado histórico y riqueza cultural. 
Cuando un niño o niña decide iniciar los estudios de música, seguramente no se plantea si hay más o menos representación de uno u otro sexo en la composición, en la dirección o en el mundo de la pedagogía musical. Sin embargo, a medida que pasan los años de estudio y sobre todo cuando se explica la historia de la música, sus orígenes (al menos lo que se conocen hasta la fecha) y su evolución, la falta de referentes femeninos sí puede ser un condicionante a la hora de elegir una u otra rama dentro de los estudios musicales.

Cuestiones como la preferencia de un estilo musical, la danza, la elección de un instrumento musical e incluso el comportamiento que se tiene en un ambiente musical, son condicionantes ${ }^{2}$ Se define estereotipo de género como el conjunto de que generan los diversos estereotipos ${ }^{2}$ de características que se atribuyen a un grupo humano género. Debido a lo anterior, es necesario (su aspecto, su cultura, sus costr factor para generalizar y categorizar a las personas, conocer los factores socioeducativos que
negando su identidad individual. influyen en la elección de un instrumento o de ciertas prácticas musicales para poder evitar los sesgos de género latentes $y$, de esta manera, crear nuevas alternativas que posibiliten un progreso ante tal situación.

En la actualidad, existen diversas iniciativas que favorecen la difusión del trabajo de mujeres compositoras, intérpretes y directoras. Una de estas interesantes iniciativas es la programación anual de conciertos y festivales de música donde se interpretan obras de autoras de siglos anteriores y actuales. 
MUJERES $Y$ DIRECCIÓN

Antes de adentrarnos en la explicación del rol del director $^{3}$ de orquesta y la situación de la mujer directora, se considera importante explicar qué es una orquesta, cuál es su origen y cuál ha sido su evolución.
${ }^{3}$ Orquesta: diccionario de la Real Academia Española, denomina a la orquesta como el "conjunto de instrumentos de cuerda y viento que tocan unidos en los teatros y en otros lugares". Según su composición, se distingue entre orquesta sinfónica, de cámara, de cuerdas...y según el género que interpretan podemos denominarlas de ópera, de música ligera, de baile...En el siglo XVII se establece una distinción clara entre música de cámara y música orquestal, esto es, músicos que tocan distintas líneas melódicas o músicos que doblan iguales partes musicales estableciendo un concepto concertante (Grout y Paliska, 1995).

\section{LA ORQUESTA Y SU EVOLUCIÓN}

En el siglo XII se fundaron los primeros gremios de músicos. Amenizar los enlaces matrimoniales, ceremonias y festividades se convirtieron en tareas profesionales para los integrantes de estos gremios. No obstante, habría que esperar hasta el siglo XVII para que los músicos se emplearan como directores de escuelas de música, organistas en alguna iglesia, músicos de corte o municipios, y finalmente se fueran incorporando a servicios militares. En Europa durante ese mismo siglo, existió una gran demanda de músicos para establecerse en las orquestas de los nobles adinerados, ya que era propio de un estatus social elevado poseer una orquesta. Además, en este periodo se celebraron innumerables festivales y eventos, que facilitaron la proliferación de las orquestas.

Posteriormente, en el siglo XVIII, se produjo una serie de recortes presupuestarios que supusieron el final de muchas or- 
questas privadas. Era frecuente que los músicos, además de pertenecer a alguna orquesta, impartieran clases, vendieran instrumentos, o bien ofrecieran conciertos de cámara (Bennett, 2010 , p. 14). A principios del período clásico europeo (1750 y 1820), la orquesta estaba compuesta por primeros y segundos violines, violas, violonchelo, un contrabajo, dos oboes, dos trompas y timbales. Progresivamente se fueron incorporando otros instrumentos de viento, como las flautas traveseras, el clarinete (introducido por Mozart hacia 1750) y el fagot. Hacia finales del siglo XVIII, Beethoven amplía la orquesta incluyendo dos trompetas y dos timbales.

Aproximadamente entre 1750 y 1800 se consolida la orquesta sinfónica en Europa. Las indicaciones sobre la orquestación en las partituras tendrían un carácter más claro y preciso. Además, se convirtió en un periodo de avances técnicos en los instrumentos, por lo que se daría una mejora en su sonido y mayores posibilidades técnicas. Mannheim y Viena fueron los centros de producción musical más importantes de aquella época. (Aster, 2012, p. 55).

Durante el siglo XIX, denominado Romanticismo, se dieron una serie de transformaciones que afectaron al conjunto orquestal. Hacia 1800, el músico comienza a disfrutar de la autonomía y poco a poco es más independiente, de este modo, ya no tiene que estar al servicio de un mecenas. El compositor deja de ser el funcionario de corte para convertirse en un artista independiente. Claros ejemplos fueron Beethoven, Schubert 
y Berlioz, quienes vivieron y murieron por y para su arte (Platinga, 1992, p. 22). A mediados del XIX comienzan a aparecer las primeras orquestas profesionales constituidas para festivales concretos, orquestas de teatro u opera, como la Filarmónica de Liverpool (1840), la Halle (1860) o la Sinfónica de Londres (1900). Actualmente la orquesta sinfónica consta de unos 6065 músicos variando en algunos casos y dependiendo del repertorio a ejecutar. Los instrumentos se seleccionan según el carácter y la instrumentación de la obra.

Las diferentes expresiones artísticas atraviesan, desde hace ya algunas décadas, momentos críticos debido a la falta de financiación y público asistente. Esta situación afecta gravemente a la música clásica y a las personas y organismos que le dan vida en un gran número de países europeos.

\section{EL MÚSICO DE ORQUESTA EN EL SIGLO XXI}

Una vez que el músico de orquesta ha finalizado sus estudios, debe decidir qué camino tomar: dedicarse a la docencia, ser instrumentista, o como es habitual, compaginar ambas facetas, ya sea trabajando en una orquesta o en una agrupación de cámara e impartiendo clases en algún centro de música.

Un tema a analizar es si desde los conservatorios o centros superiores de música, se dota al alumnado de herramientas y habilidades suficientes para adaptarse a su futuro laboral. Fre- 
cuentemente, los jóvenes acceden a los conservatorios superiores para formarse como instrumentistas sin ni siquiera conocer las salidas profesionales que los diferentes estudios musicales pueden brindarles. (Bennett, 2010, p. 55).

La labor que realizan las orquestas actuales, a diferencia de las orquestas de siglos pasados, es tratar de incluir una función interpretativa, y la realización de proyectos de carácter social y educativo, tal y como lo hacen en países como España. Para ello, es necesaria una reflexión sobre la figura del músico, imprimiéndole un carácter más flexible y un perfil más completo y multifuncional. Es necesario, por tanto, adaptar el perfil de nuestros futuros músicos profesionales a las necesidades que las orquestas demandan. Como expone Bennett,

a medida que las orquestas asumen un papel educativo y comunitario mayor, es fundamental que los músicos dominen las habilidades que les permitan interactuar satisfactoriamente con esos programas. Parece ser que muchas organizaciones entienden que faltan recursos para dicha formación. (Bennett, 2010, p. 71)

La concepción romántica que consideraba que los buenos directores nacen y no se hacen ha sido refutada por múltiples estudios actuales. Por tanto, partimos de la base de que un 
director/a de orquesta debe poseer ciertas habilidades, además de una sólida formación académica. Debido al crecimiento de los componentes de las agrupaciones musicales y al progresivo abandono del bajo continuo, realizado por el clavicémbalo en la mayoría de los casos, se instauró la figura del director de orquesta (Gustems y Elgstrom, 2008, p. 68).

\section{DIRECCIÓN Y LIDERAZGO}

Como seres sociales que somos, nos organizamos, ello implica una coordinación y organización social, y en este contexto la figura de un líder es fundamental. El vocablo líder proviene de la lengua inglesa "to lead", es decir, guiar. (Molero, 2009, p. 23). Así entonces, en un grupo de personas independientemente del sexo y del número de éstas, el líder es quien se encarga de guiar a las demás que dependen de él/ella. En ellas encontraremos seres sociales que son líderes y otros que son subordinados.

La presencia de líderes está en muchos y muy variados ámbitos: en el político, el deportivo, el educativo, el mundo empresarial. El ámbito musical no es una excepción. La gestión interna de una orquesta es un claro ejemplo de ello. Así pues, los miembros de una orquesta son una organización que está compuesta por varios músicos altamente cualificados que siguen las instrucciones de un líder. En este caso el líder es el director/a de orquesta. 
Los estudios de liderazgo parten de la existencia de, al menos, una persona que siga a dicho líder y, algún tipo de ritual que lo acredite como tal (Peterson y Seligman, 2004, p. 89). Al situar el liderazgo como una competencia social, podemos decir que hablar de liderazgo es hablar de integridad, autoridad, poder, dominio, asertividad social (Bass, 1985, p. 106).

Un líder es capaz de enfrentarse a una situación compleja, así como resolver conflictos, tomar la palabra en nombre del grupo al que representa, tomar la iniciativa en circunstancias sociales e implicarse en situaciones de emergencia o peligro. Según Goleman (Goleman, 2014, pp. 25-26) los líderes marcan estrategias, motivan, crean una misión, implantan una cultura.

Nuestra sociedad se transforma a diario y muy rápidamente. En este proceso de cambio, la mujer deja atrás su rol secundario del mundo privado que la ha mantenido alejada del mundo público. De esta manera, la figura femenina del siglo xx y XXI ha ido incorporándose a la población económicamente activa, ha podido mejorar su calidad de vida, se ha integrado al sistema educativo y en general ha alcanzado un lugar de relevancia en la sociedad. No obstante, persisten todavía prácticas y discursos que fomentan la dicotomía femenino/ masculino en torno a la actividad de liderazgo. Así, todavía hoy permanece la imagen de hombre arquetipo maestro/director (estereotipo líder-macho). Expone la crítica musical McClary: 
La confusión sobre si la música corresponde a la mente o al cuerpo se intensifica cuando la oposición binaria fundamental entre masculino y femenino se proyecta sobre ella. En la muy importante medida en que la mente se define como masculina y el cuerpo como femenino en la cultura occidental, la música corre siempre el riesgo de que se la considere como un asunto completamente femenino, y uno de los medios de afirmar el control masculino del medio consiste en negar la misma posibilidad de la participación de las mujeres, porque icómo puede ser femenino un asunto si se excluyen de él a las mujeres de carne y hueso? (1994, pp. 95-98)

A lo largo del pasado siglo xx, aparecieron las teorías del liderazgo paulatinamente. A grandes rasgos podemos decir que las cuatro tipologías más destacadas son:

- Liderazgo autocrático: el líder lo establece todo y es el encargado de otorgar recompensas o castigos (poder personal absoluto).

- Liderazgo democrático: el líder procura implicar en la toma de decisiones a los subordinados. - $\quad$ Liderazgo liberal: (también denominado laissezfaire) el líder otorga libertad total a los integrantes del grupo sin intervenir. 
- $\quad$ Liderazgo transformacional: se cuestiona cómo transforman los/as líderes las organizaciones.

Motivación, independencia, tolerancia a la ambigüedad, interés por la participación y experiencia, capacidad perceptiva y creación del estímulo adecuado, son necesarias para que una directora-líder pueda desarrollar su trabajo.

La presencia de mujeres ocupando la posición de líder, coordinadora y/o guía es más bien escasa e incluso inexistente en muchos ámbitos. Volviendo al ejemplo anterior, ha sido impensable durante cientos de años que la figura del director de una orquesta sea una mujer. Hasta tiempos recientes, el mundo de la música clásica ha estado dominado por el sexo masculino. Los condicionantes de género son perpetuados a lo largo de la historia a través de los procesos de socialización.

Es fundamental en los diversos contextos educativos donde se transmiten o difunden los códigos de género que cada sociedad o grupo cultural impone, entendidos estos como las reglas sociales que marcan el significado de lo que se considera masculino o femenino. (Flecha y Giroux, 2001, pp. 25-27)

Otro de los principales motivos por los cuales ha sido tan difícil que la mujer llegue a ocupar el cargo de directora de 


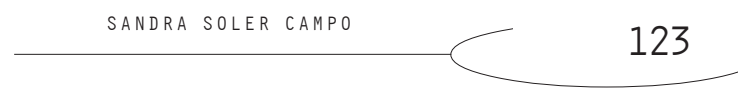

orquesta es el denominado techo de cristal ${ }^{4}$. Es decir, la barrera transparente que no permite a la mujer acceder a cargos directivos o coordinación por el simple hecho de ser mujer. El sexo femenino ${ }^{4}$ Techo de cristal: barrera invisible resultante de un complejo entramado de estructuras en organizaciones dominadas por varones, que impide que las mujeres accedan a puestos importantes. (100 palabras para la igualdad. Glosario de términos relativos a la igualdad entre hombres y mujeres (1998), Madrid: Comisión Europea, Dirección General de Empleo, Relaciones Laborales y Asuntos Sociales).

ha tenido que realizar, y continúa haciéndolo, un intenso esfuerzo para poder superar una serie de barreras sociales y culturales.

Lupano y Castro añaden que las mujeres que llegan a ser "líderes" pueden ser calificadas negativamente debido a dos motivos:

- Porque no ponen en marcha las características que las personas comúnmente relacionamos con el liderazgo.

- En caso de desplegarlas, son consideradas muy poco femeninas.

El hecho de que las mujeres no "encajan"en los moldes de líder estereotipo da lugar a que aquellas que quieren ser líderes necesitan normalmente estar extremadamente bien cualificadas, demostrar que son muy buenas en esa ocupación y estar muy preparadas para dicho puesto (Castro y Lupano, 2005).

Uno de los desafíos de las organizaciones del futuro es aceptar una variedad de estilos de liderazgo. No existe el "mejor" es- 
tilo de liderar, todo dependerá de la organización y del tipo de trabajo a realizar. Tanto hombres como mujeres deberían sentirse libres de adoptar las estrategias de liderazgo que consideren más adecuadas en cada caso. El reconocimiento de tal diversidad de estrategias va a hacer posible que las personas que se encarguen de la gestión de empresas, sin importar el sexo al que pertenezcan no se vean obligadas a repetir patrones de liderazgo que en décadas pasadas se asociaban al sexo masculino y/o femenino, sino que según sus habilidades, cualidades, destrezas y objetivos a conseguir actuará de un modo u otro. En la actualidad, no podemos negar que está presente la dicotomía femenino/masculino en torno a la actividad de liderazgo, y consecuentemente a la de director/a de orquesta.

\section{LA FIGURA DEL DIRECTOR/A}

En general, la figura del director/a de orquesta es a menudo representada con cierto estatus y poder absoluto. A menudo se la presenta como un/a artista inspirado/a, dedicado/a y comprometido/a con la música y encargado/a de dirigir la orquesta con una mano "maestra". No obstante, no todos los directores/as imponen el mismo grado de autoridad sobre los músicos a los que dirige. Esta visión excluye cualquier circunstancia externa que puede dañar su estatus autoritario, ejemplo de esto son las dificultades que puede llegar a tener un director/a con ciertos músicos o sectores de 


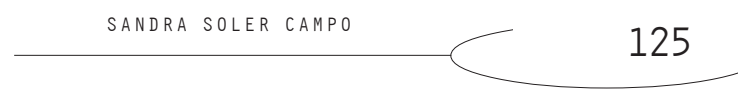

músicos o el saber adaptarse a una orquesta ya establecida institucionalmente.

En la actualidad, la figura del director/a de orquesta parece ser un constructo social que se crea detrás de los escenarios durante los ensayos. Dicha figura ha adquirido gran notoriedad en las últimas décadas. En algunos casos, directores como Berstein, Von Karajan o Baremboim, han estado rodeados de un poderoso entorno mediático. Éste último, Baremboim, expone que "La dirección de orquesta como actividad única es una invención sociológica, no artística, del siglo $x x^{\prime \prime}$.

${ }^{5}$ Diario digital El País, publicado el 29 de junio de 2007. Recuperado de: https://elpais.com/diario/2007

La primera orquesta documentada de la historia fue la del rey Saúl en 1025 a. C., la cual tenía veinticuatro instrumentistas de viento y cuerda. En la Antigua Grecia, durante las prácticas musicales, los intérpretes seguían los movimientos del intérprete principal. En la Europa medieval, los maestros de coro empezaron a marcar el tempo con un bastón corto. Los grupos instrumentales barrocos seguían al primer violín y Claudio Monteverdi (1567- 1643) crearía en Venecia el título de Maestro de Capilla, este se encargaría de coordinar a los músicos para que toquen juntos. En Alemania, a esta figura se le denominaba Kapellmeister. En el siglo XVII el responsable del bajo continuo dirigía desde el teclado la agrupación y, normalmente, era el Kapellmeister, quien además organizaba los ensayos del grupo (Lebrecht, 1997). 
A lo largo del siglo XVIII se desplaza la importancia que tenía el teclado durante el siglo anterior, y toma su relevo el primer violinista. Éste se posiciona frente a la orquesta, lo que le daba suficiente visión del conjunto y además le permitía tener un buen contacto visual con los músicos. Este modo de dicción se continuó utilizando en óperas y en música de concierto durante el siglo XVIII e inicios del XIX en países como Inglaterra, Alemania e Italia.

A Louis Spohr (1784-1859) se le atribuye el inicio del uso de la batuta en abril de 1820. Desde mediados del siglo XIX, sería habitual utilizar batuta de madera para dirigir. No obstante, continuarían permaneciendo unidas las figuras de director y compositor. Berlioz fue el primer compositor en considerar la dirección orquestal como un arte en sí. En 1844 publicó su Grand Traite d'Instrumentation et d'Orchestration Modernes, al que amplió un capítulo en 1855 sobre la dirección orquestal. En la segunda mitad del siglo XIX la profesión de director se desarrolla independientemente y fue adquiriendo importancia. El primer director considerado profesional será Hans Von Bullow (Bowen, 2005, pp. 33-37).

Es durante el momento del ensayo cuando el director/a intenta trascender la relación contractual para poder establecer una legitimidad profesional. Dicha legitimidad, es construida a través de la interacción y puede ser invalidada en cualquier momento. A grandes rasgos podemos establecer tres compo- 
nentes sobre los cuales se basa la legitimidad profesional (Wallis, 1984, pp. 34-38).

- Dominio de la actividad que se está llevando a cabo. Aquí se incluye también la precisión de sus gestos, ya que es en sí misma una forma de autoridad.

- Interpretación musical con fundamento. El director tiene que ser capaz de explicar de modo coherente por qué ha decido interpretar de una manera determinada un pasaje de la obra. Es decir, explicar y/o justificar el porqué de su interpretación personal de la pieza musical. El objetivo principal sigue siendo la adherencia de la orquesta.

- Destreza para gestionar al grupo: la orquesta. No todos los directores/as de orquesta logran una comunicación y cohesión fluida con la orquesta. El caso de muchos directores invitados es un claro ejemplo.

El director/a podrá ser más o menos autoritario, ello dependerá por una parte de su personalidad, y por otra, de factores como la respuesta de los músicos de la orquesta hacia él/ella y/o el ambiente que haya habido durante los ensayos previos a la actuación. Las interacciones realizadas por las palabras y los gestos (tanto interacciones verbales como no verbales, 
por ejemplo, gestos, compromiso corporal, expresiones, etc.) son información de suma importancia tanto para el emisor (director) como para los receptores (músicos y audiencia).

Según Delalande (2008), las diferentes interacciones iniciadas por un director/a de orquesta durante un concierto desde su llegada al escenario, es decir, los aplausos iniciales, los gestos y la mímica realizados durante la actuación, determinarán sin duda alguna el éxito o fracaso de éste. Es el director/a quien se encarga de levantar la batuta e indicar a los músicos el inicio de la obra, además de designar el tempo y las dinámicas. Con la mano derecha e izquierda va indicando cuando entra un instrumento o conjunto instrumental, cuando entra un segundo grupo, cuando deja de tocar el primero, cuando debe intervenir un solista. Por ello, el conocimiento de la obra musical por parte del director debe ser muy profundo.

Además de los gestos, hay dos elementos claves en el intercambio de información que se produce durante la ejecución instrumental, bien sean en los ensayos, o en el momento del concierto mismo, estos son: la escucha y el contacto visual. Si la escucha entre un director y los músicos es buena, y si los músicos tienen experiencia, entonces la respiración fisiológica corresponde a la articulación del discurso musical. En determinados repertorios musicales, la elección de distintos tipos de respiración en uno o varios pasajes darán lugar a discursos musicales diferentes. 


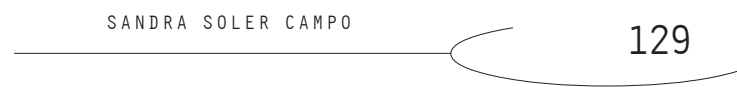

MUJERES DIRECTORAS DE ORQUESTA

El papel del/la director/a de orquesta tiene una gran responsabilidad, pues tiene bajo el mando de su batuta a decenas de personas que siguen sus "órdenes", debido a ello, se ha utilizado muchas veces como símbolo de poder absoluto. Éste es uno de los motivos que explica por qué el número de mujeres que se dedican a esta profesión es menor. Antes de que una orquesta interprete una obra musical para un público, el hecho de que la persona que dirija a los músicos sea un hombre o una mujer no deja indiferente a nadie. Esto se debe a la escasa presencia de mujeres en esta profesión (Ravet, 2006, pp. 58-65).

En el marco de la dirección orquestal, a diferencia de la dirección coral, el uso de la batuta es vista como un indicador de virilidad y de deseo por dar órdenes a un conjunto de músicos. Las burlas y comentarios discriminatorios hacia la mujer directora de orquesta por parte de los músicos, sobre todo hombres que forman parte de ella, se dan en ocasiones para conocer cuál es su reacción y nivel de autoridad.

En el ámbito de la dirección orquestal, el dominio masculino es un hecho evidente. Así, la mayoría de los estudiantes de dirección de los Conservatorios Superiores en España, pertenecen al sexo masculino ${ }^{6}$. La presencia de mujeres que estudian dirección orquestal es todavía en la actualidad 
escasa, incluso en algunas Escuelas Superiores de Música no hay ninguna mujer matriculada en alguna promoción.

La aportación femenina en el mundo de la dirección orquestal es un fenómeno relativamente nuevo. A pesar de que contamos con algunos antecedentes aislados como el de la vienesa Marie Grunier en el siglo XIX, no será hasta la segunda mitad del siglo xx cuando contaremos con mayor presencia de mujeres directoras de orquesta. En la primera mitad del pasado siglo xx, no se concibe la presencia femenina "en el podio". En un ambiente donde los directores solían ser líderes autoritarios, la mujer no tenía cabida. Incluso dos de las filarmónicas más importantes de Europa, la de Berlín y Viena, hasta tiempos recientes no aceptaban que una mujer formara parte de su plantilla instrumental. Es gracias en gran parte a la revolución feminista de los años 60 que la integración de la mujer en todos los ámbitos es más favorecedora.

Los estudios de dirección orquestal en los conservatorios de nuestro país no pudieron realizarse oficialmente hasta el año 1969 en Madrid. A pesar de varios intentos y teniendo en cuenta que la reforma educativa de 1942 contempló la implantación de dicha especialidad en el currículo de los conservatorios. Se deduce que todas aquellas personas interesadas en estudios de composición, tanto hombres como mujeres antes del año 1969, aprendían mediante clases privadas o marchando al extranjero aquellos quienes tenían un elevado poder adquisitivo. 
En España no se impidió que la mujer accediera a los estudios de orquesta durante la segunda mitad del siglo xx. En otros países de la actual Unión Europea, ocurría totalmente lo contrario. Así pues, una mujer no podía realizar este tipo de estudios musicales (aunque sí podía estudiar piano, violín, arpa, canto...), sin embargo, esta situación que en principio parece favorecedora para la mujer debe conocerse a fondo. Si bien la mujer tenía libre acceso a estudiar dirección orquestal, no podía escoger las mismas asignaturas que un hombre. Su formación académica se limitaba a estudiar piano, declamación y materias que le enseñaran a ser una "señorita"capaz de hacer lo que la sociedad espera de ella, es decir, las tareas del hogar con buenos modos. Las materias de contrapunto, fuga y composición eran a las que accedían aquellos hombres que ingresaban a estos estudios (materias imprescindibles para adentrarse en el complejo mundo de la técnica de la composición y/o dirección orquestal).

Por ello, negar el acceso de la mujer a estas materias de tan importante peso en los estudios de dirección, era sinónimo de impedimento y discriminación. Como en la mayoría de los casos, existen excepciones, en España debemos destacar la vida y obra de Elena Romero, quien de modo autodidacta se inició en el mundo de la dirección orquestal cosechando grandes éxitos dentro y fuera de nuestro país, obteniendo en el año de 1953 el prestigioso Premio de Composición Felip Pedrell. Podemos decir que fue ella la primera mujer que dirigió en 
este país una orquesta sinfónica. A pesar de haber roto moldes, al estar constantemente rodeada de hombres, y siendo una "pionera"y referente para otras mujeres que quisieran dedicarse a este campo, Elena nunca se posicionó al lado del feminismo radical.

Son muchas las situaciones en las que la mujer directora ha tenido que luchar por su dignidad y por poder desarrollar su trabajo con cierta normalidad. Citaré dos ejemplos que creo son dignos de comentar en este apartado. La directora Simone Young, primera mujer a la que se le permitió dirigir la Filarmónica de Viena, tuvo que discutir con la administración de la Filarmónica de Hamburgo por poder dirigir una sinfonía de Antonio Bruckner y no solo piezas sinfónicas de Mozart.

Por otra parte, la directora de orquesta Marin Alsop, inició por la vía judicial una ardua lucha por poder llegar a ser directora permanente con la orquesta sinfónica de Baltimore. Finalmente ganó la batalla y pudo dirigir la

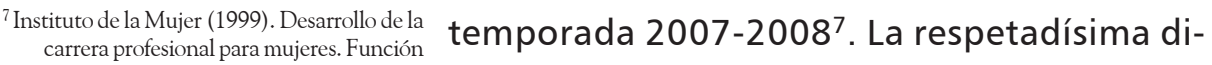
directivayliderazo. rectora de orquesta española Isabel López Calzada, directora de la Orquesta de Mujeres de Madrid, desarrolla su labor artística entre el pasado siglo xx y el actual siglo XXI.

En España se constituyó en 2012 la Asociación de Directores de Orquesta (AESDO), la cual está formada tanto por hombres como mujeres directores/as de orquesta. Su principal objetivo es el de apoyar e impulsar el desarrollo de dicha 


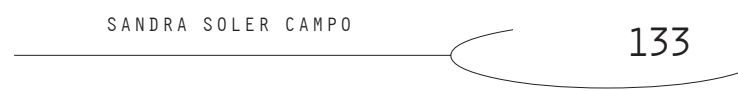

profesión y la transmisión de conocimiento a la sociedad. Es importante la aparición de estas asociaciones para poder estar unidos y caminar en una misma dirección. Es curioso que, desde su reciente creación, de los 44 miembros asociados tan sólo cuatro sean mujeres. En este sentido, Lara Diloy, directora asistente de la Barbieri Symphony Orchestra, expone: "Creo que desde la asociación también debemos implicarnos en la labor de difusión del trabajo que estamos haciendo las mujeres en la Dirección de Orquesta, con objeto de normalizar la profesión como algo tan masculino como femenino"(2013, p. 53).

La socióloga y musicóloga de origen francés Hyacinthe Ravet, tras la realización de un estudio basado en la observación de una misma orquesta dirigida por el director Claudio Abbado y por la directora Claire Gibault, concluye que cuando una orquesta es dirigida por una mujer, ésta debe hacerse valer y esforzarse mucho más que un hombre para que los músicos acepten su autoridad. Además, expone la autora: "en general, los modos de desestabilización de las mujeres directoras y las burlas y chistes hacia éstas por parte de músicos (sobre todo hombres) se dan con mayor frecuencia hacia ellas que hacia el sexo masculino"(Ravet, 2006, pp. 82-96).

En la actualidad, todavía se duda acerca de las capacidades que pueda tener una mujer a la hora de dirigir una orquesta. La crítica y la prensa cuestionan con frecuencia temas como la programación y la interpretación, exponiendo que las mu- 
jeres tan sólo son capaces de dirigir "música de mujeres" o "pequeñas" obras y no "grandes sinfonías"románticas o postrománticas, y lo que es peor todavía, se afirma que las mujeres dirigen de un modo "diferente". Otro de los tópicos y comentarios recurrentes en la prensa, es presentar a la mujer directora como algo exótico, como curiosidad y como una excepción (Schweitzer, 2010, pp. 54-56).

Entre los diez directores de orquesta más reconocidos a nivel mundial no se encuentra en la actualidad ninguna mujer. Las escasas ocasiones que ha habido alguna mujer en este ranking, no ha llegado a tener una posición permanente. Esta situación no es una excepción en nuestro país, son muchas las mujeres que deciden realizar en España los estudios de composición. En el capítulo dedicado al análisis de las entrevistas que se han realizado, puede apreciarse que hay un número importante de mujeres que se dedican a la dirección.

\section{CONCLUSIONES}

Una buena formación musical debe comenzar a una edad temprana. Lo ideal sería, aunque quizás suene utópico, hacer de la música una lengua materna, y hacer que este aprendizaje sea vital. En cada etapa de nuestra vida, como es lógico, nos acercaremos a nuevos aprendizajes. Tener un espíritu crítico hacia ellos es importante, además de ser capaces de discriminar la buena de la mala música más allá de cuestiones de género. 
Parece importante que las nuevas generaciones sean capaces de apreciar críticamente los elementos de interés expresivo y estético y desarrollar criterios propios de evaluación, al igual que conocer y respetar las principales manifestaciones artísticas presentes en el entorno.

Del mismo modo, es necesaria una formación en cuestiones de género del profesorado en los diferentes grados de enseñanza: educación primaria, educación secundaria y superior, estudios universitarios, grados de conservatorio. Para ello, es importante también que los manuales, libros de texto, enciclopedias, partituras, material sonoro y visual cuando sea posible, así como páginas web incluyan a estas mujeres. Es necesario e imprescindible que los docentes y personas encargadas en el proceso de enseñanza y aprendizaje de los futuros estudiantes transmitan valores que respeten la igualdad de género y sean capaces de "re-mirar" nuestro pasado y presente de modo crítico, incluyendo personas de ambos sexos que hayan destacado en el mundo de la composición, de la dirección y de la interpretación.

\section{Bibliografía}

Aster, M. (2012). La Orquesta del Reich. Barcelona: Edhasa.

BAss, B. (1985). Leadership and Performance beyond expectations. New York: The Free Press.

Bennett, D. (2010). La música clásica como profesión. Pasado, presente y estrategias para el futuro. Barcelona: Editorial Graó. 
BOWEN, H. (2005). ¿Does the music matter? Motivations for attending a music festival. New York: Cognizant Communication Corporation.

CAstro, A. y LUPANO, M. L. (2005). Diferencias individuales en las teorías implícitas del liderazgo y la cultura organizacional percibida. Boletín de Psicología, (85), 89-109.

Delalande, F. (2008). Un millón de compositores. En A. Giraìldez (Coord.), Percepción y expresión en la cultura musical básica (pp. 7-17). Madrid: Ministerio de Educación, Política Social y Deporte.

DiloY, L. (noviembre 2013). Ganarse el respeto. "La musa invisible". Actas de las Jornadas sobre música y mujeres. Madrid: Conferencia llevada a cabo en el Real Conservatorio Superior de Música de Madrid.

FlechA, R. y GiRoux, H. A. (2001). Cultura, política y práctica educativa. Barcelona: Editorial Graó.

Goleman, D. (2014). Liderazgo. El poder de la inteligencia emocional. Barcelona: B de Bolsillo.

Grout, D. y Palisca, C. (1995). Historia de la Musica Occidental (Vol I). Madrid: Alianza.

Gustems, J. y ElgStRÖM, E. (2008). Guía Práctica para la dirección de grupos vocales e instrumentales. Barcelona: Graó Biblioteca de Eufonía.

INSTITUTO DE LA MUJeR. (1999). Desarrollo de la carrera profesional para mujeres. Función directiva y liderazgo (1999). Madrid: Instituto de la Mujer.

Lebrecht, N. (1997). ¿Who killed Classical Music? Maestros, Managers and Corporate Politics. Manchester: Birch Lane. 


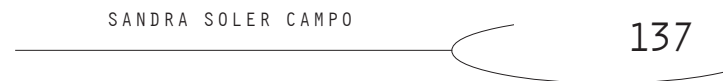

MCCLARY, S. (1994). Feminine Endings. Music, Gender and Sexuality. Minessota: University of Minnesota Press.

Molero, F. (2009). Mujer y liderazgo en el siglo xxı. Madrid: Instituto de la mujer.

Peterson, C. y Seligman, M. E. P. (2004). Character strengths and virtues: A handbook and classification. New York: Oxford University Press and Washington DC: American Psychological Association.

Platinga, L. (1992). La Música Romántica. Una historia del estilo musical en la Europa decimonónica. Madrid: Akal.

RATtLE, S. (junio de 2007). Entrevista: Simón Rattle. La batuta más envidada del mundo. [Impresa]. Recuperado de https://elpais.com/diario/ 2007/07/01/eps/1183270554_850215.html

RAVET, H. (2006). L'orchestre au travail. Interactions, négociations, coopérations. Paris: Vrin.

SChWeitzer, S. (2010). Femmes de pouvoir: unes histoire de l'égalité professionnelle en Europe. París: Payot.

Wallis, B. (1984). Arte después de la Modernidad. Nuevos planteamientos en torno a la representación. Madrid: Akal. 